\title{
A GEOMETRIC INTERPRETATION OF A CLASSICAL GROUP COHOMOLOGY OBSTRUCTION
}

\author{
R. O. HILL, JR.
}

\begin{abstract}
For a non-Abelian group $G$, we show that the obstruction to the existence of an extension of $G$ by $\Pi$ that induces $\phi: \Pi \rightarrow$ Out $G$ is also the $k$-invariant of the classifying space for $K(G, 1)$-bundles.
\end{abstract}

1. In the classical theory of group extensions, there arises a group cohomology obstruction. This paper studies some topological implications of that obstruction, giving it a geometric interpretation and augmenting the classification of fibrations by J. Siegal [15], [16] with the case the fiber is a $K(G, 1), G$ not Abelian.

If $1 \rightarrow G \rightarrow E \rightarrow \Pi \rightarrow 1$ is an extension of the group $G$ by the group $\Pi$, it induces either an action $\phi: \Pi \rightarrow$ Aut $G$, if $G$ is Abelian, or a "semiaction" $\phi: \Pi \rightarrow$ Out $G=$ Aut $G /$ In $G$, otherwise. See [9, p. 124]. Given $G$ not Abelian and a $\phi: \Pi \rightarrow$ Out $G$, there need not exist an extension inducing $\phi$. Indeed, let $C$ be the center of $G$ and $\alpha$ : Out $G \rightarrow$ Aut $C$ be induced by restriction Aut $G \rightarrow$ Aut $C$. By [4] or [9, p. 128] we have

Theorem A. There is an obstruction $k \in H_{\alpha \phi}^{3}(\Pi ; C)$ to the existence of an extension of $G$ by $\Pi$ which induces $\phi$. If $k=0$, the set of all equivalence classes of all such extensions is in a 1-1 correspondence with $H_{\alpha \phi}^{2}(\Pi ; C)$.

In $\$ 4$ this obstruction is given in detail, and we prove the following, which shows this obstruction has a "universal example."

THEOREM B. Let $U \in H_{\alpha}^{3}$ (Out $G ; C$ ) be the obstruction to the existence of an extension of $G$ by Out $G$ which induces id: Out $G \rightarrow$ Out $G$. Then for any $\phi: \Pi \rightarrow$ Out $G, \phi^{*}(U)$ is the obstruction $k$ of Theorem A.

Recall, even though $K(G, 1)$ is not an $H$-space (for $G$ not Abelian), there is a universal classifying fibration $K(G, 1) \rightarrow E \stackrel{p}{\rightarrow} B$. By Gottlieb [6, p. 54], $\pi_{1}(B) \cong$ Out $G, \pi_{2}(B) \cong C$ and $\pi_{i}(B)=0$, otherwise. Thus $B$ has a single (twisted) $k$-invariant in $H^{3}(K($ Out $G, 1) ;\{G\})$ (where \{\} denotes local coefficients). We denote by $\Phi: H_{\alpha \phi}^{*}(\Pi ; C) \rightarrow H_{\alpha \phi}^{*}(K(\Pi, 1) ;\{C\})$ the natural isomorphism (see [9, IV. 11]). The main result of this paper is

TheORem C. Let $U$ be given by Theorem B. Then $\Phi(U)$ is the (twisted) $k$ invariant for the universal classifying space for $K(G, 1)$ bundles.

Theorem A has a natural geometric consequence. Given an extension $G \stackrel{i}{\rightarrow} E \stackrel{p}{\rightarrow} \Pi$, we can construct a fibration $K(G, 1) \stackrel{i^{\prime}}{\rightarrow} K(E, 1) \stackrel{p^{\prime}}{\rightarrow} K(\Pi, 1)$

Received by the editors November 1, 1974.

AMS (MOS) subject classifications (1970). Primary 55F15; Secondary 55G35, 18H10, $20 \mathrm{~F} 25$. 
in which $i_{*}^{\prime}=i$ and $p_{*}^{\prime}=p$ on the fundamental groups. In any fibration, "dragging" the fiber around loops in the base (backwards) induces an action of $\pi_{1}$ (base) on $\pi_{n}$ (fiber), but only modulo the action of $\pi_{1}$ on $\pi_{n}$. Thus, the fibration $p^{\prime}$ induces a geometric "semiaction" $\pi_{1}$ (base) $\rightarrow$ Out $\left(\pi_{1}\right.$ (fiber) $)$ in addition to the algebraic one.

THEOREM D. These two "semiactions" are the same.

This may be proven by extending either the proof of Theorem 1(a) in [7] or [14, pp. 86-88]

Using $\Phi$ we therefore easily get as a corollary of Theorem A:

Theorem E. Given $a \phi: \Pi \rightarrow$ Out $G$, there is an obstruction

$$
k \in H_{\alpha \phi}^{3}(K(\Pi, 1) ;\{C\})
$$

to the existence of a $K(G, 1)$-fibration over $K(\Pi, 1)$ which induces $\phi$. If $k=0$, the set of all equivalence classes of such fibrations is in a 1-1 correspondence with $H_{\alpha \phi}^{2}(K(\Pi, 1) ;\{C\})$.

Although Theorem A gives a proof of this, it does not give any geometrical insight to this geometric fact. Theorem $\mathrm{C}$ leads to a very satisfactory geometric proof, which is given in $\S 3$.

The proof of Theorem $\mathrm{C}$ yields a complete description of $p$.

Theorem F. Let $K(G, 1) \rightarrow E \stackrel{p}{\rightarrow} B$ be the universal $K(G, 1)$-fibration. Then $E$ is a $K$ (Aut $G, 1)$, its homotopy sequence reduces to the natural $0 \rightarrow C \rightarrow G$ $\rightarrow$ Aut $G \rightarrow$ Out $G \rightarrow 1$, and the "semiaction" of $\pi_{1}$ (base) on $\pi_{1}$ (fiber) corresponds to the identity: Out $G \rightarrow$ Out $G$.

Theorems $\mathrm{C}$ and $\mathrm{F}$ follow from 3.1 and 3.2 .

I would like to thank Professor William Massey who first posed to me the problem of geometrically understanding Theorem A.

I understand recently that some of the above with somewhat different proofs have been known to M. G. Barratt.

As a matter of convenience (see the second half of [3]), the remainder of this paper is done in the category of semisimplicial complexes. The reader is assumed to be familiar with May [10], as notation tends to be based on that text.

2. We give a concise development of $L_{\pi}(C, n)$ and its classifying properties. For more details see [5], [8], [11], [12], [13], [15], [16], [17].

We will denote an Eilenberg-Mac Lane complex of type $(C, n)$ by $K(C, n)$, its "universal class" in $H^{n}(K(C, n) ; C)$ by $V$ and the universal principal "looppath" fibration by $K(C, n-1) \rightarrow L(C, n) \stackrel{P}{\rightarrow} K(C, n)$. Let $W(\Pi)$ denote the standard free acyclic semisimplicial complex corresponding to the group $\Pi$. If $\Pi$ acts on $C$ via $\phi: \Pi \rightarrow$ Aut $C$, then $\Pi$ acts on both $K(C, n)$ and $L(C, n)$ naturally. Using the diagonal action, we denote $W(\Pi) \times K(C, n) / \Pi$ by $L_{\pi}(C, n)$ and $W(\Pi) \times L(C, n) / \Pi$ by $T_{\pi}(C, n)$, and obtain from $P$ a Kan fibration $K(C, n-1) \rightarrow T_{\pi}(C, n) \stackrel{p}{\rightarrow} L_{\pi}(C, n)$. (The inclusion of $W(\Pi) \times$ * into $W(\Pi) \times K(C, n)$ induces a natural $K(\Pi, 1)$ contained in $L_{\pi}(C, n)$.) 
TheOREM 2.1. Let $n \geq 2$. (1) $\pi_{i}\left(L_{\pi}(C, n)\right)$ is $\Pi$, if $i=1 ; C$, if $i=n ; 0$, otherwise; and the action of $\pi_{1}$ on $\pi_{n}$ is $\phi$.

(2) There is a universal class $v \in H_{\phi}^{n}\left(L_{\pi}(C, n),\{C\}\right)$, where this denotes cohomology with local coefficients twisted by $\phi$. If $X$ is a semisimplicial complex, then $f \leftrightarrow f^{*}(v)$ is a 1-1 correspondence between the set of based homotopy classes of maps from $X$ into $L_{\pi}(C, n)$ which induce

$$
\beta: \pi_{1}(X) \rightarrow \pi_{1}\left(L_{\pi}(C, n)\right) \text { and } H_{\phi \beta}^{n}(X ;\{C\}) .
$$

(3) $A$ map $f: X \rightarrow L_{\pi}(C, n)$ lifts if and only if $f^{*}(v)=0$ if and only if $f$ is homotopic rel base point to a map whose image is in $K(\Pi, 1)$. If $f^{*}(v)=0$, the set of homotopy classes of such liftings is in 1-1 correspondence with $H_{\phi \beta}^{n-1}(X ;\{C\})$.

Parts (1) and (2) are proved by Gitler [5], and part (3) is essentially proven in Nussbaum [11], [12] and Siegel [16].

The space $L_{\text {Aut } C}(C, n)$ is understood to have the natural action of $\pi_{1}$ on $\pi_{n}$.

Suppose a Kan complex has two nonzero homotopy groups, $\pi_{1}(X)=\Pi$, $\pi_{n}(X)=C$, and $\pi_{1}$ acts on $\pi_{n}(X)$ with $\phi: \Pi \rightarrow$ Aut $C$. Then $X$ can be constructed, up to homotopy, as the pullback from the fibration $p$ by a map $f: K(\Pi, 1) \rightarrow L_{\text {Aut } C}(C, n+1)=L$ such that $\phi=f_{*}: \pi_{1}(X) \rightarrow \pi_{1}(L)$.

Definition. The element $f^{*}(v) \in H_{\phi}^{n+1}(K(\Pi, 1) ;\{C\})$ is the (twisted) $k$ invariant of $X$.

As a simple illustration, we observe

Proposition 2.2. Let $n \geq 2$. The complex $X$ is a $L_{\pi}(C, n)$ if and only if its twisted $k$-invariant is 0 .

Proof. Exercise. See Olum [13] or Robinson [17]. The case $n=1$ is treated in [7].

COROLlary 2.3. The universal classifying space for $K(G, 1)$ bundles is a $L_{\text {Out } G}(C, 2)$ iff the universal example $U=0$.

3. We prove Theorems $\mathrm{C}$ and $\mathrm{F}$ and give a geometric proof of Theorem $\mathrm{E}$. Let $A K(G, 1)$ be the semisimplicial group complex of all automorphisms of $K(G, 1)$ and let $A K(G, 1) \rightarrow W \stackrel{q^{\prime}}{\longrightarrow} B A K(G, 1)=B$ be its universal principal fibration. Then the associated $K(G, 1)$ bundle

$$
A K(G, 1) \times_{A K(G, 1)} K(G, 1) \rightarrow W \times_{A K(G, 1)} K(G, 1) \rightarrow B
$$

is the universal $K(G, 1)$ fibration $K(G, 1) \rightarrow T \stackrel{q}{\longrightarrow} B$. Compare with $[1,5.6]$. By Gottlieb [6], $\pi_{i}(B)$ is Out $G$, if $i=1 ; C$, if $i=2$; and 0 , otherwise (where $C$ is the center of $G$ ). Essentially, since the homomorphism induced on the fundamental groups by the map $A K(G, 1) \times K(G, 1) \rightarrow K(G, 1)$ is addition $C \times G \rightarrow G$, the homomorphism $\partial: \pi_{2} \rightarrow \pi_{1}$ in the homotopy sequence for $q$ "is" the inclusion of $C$ into $G$. Thus $T$ is a $K(, 1)$ and the homotopy sequence for $q$ reduces to $0 \rightarrow C \rightarrow G \rightarrow \pi_{1}(E) \rightarrow$ Out $G \rightarrow 1$. In $\S 7$ we will prove the following: 
THEOREM 3.1. There is a Kan fibration $K(G, 1) \stackrel{i}{\rightarrow} E \stackrel{p}{\rightarrow} B$ with the properties:

1. The long exact homotopy sequence for $p$ is all zero except for $0 \rightarrow \pi_{2}(B)$ $\rightarrow \pi_{1}(K(G, 1)) \rightarrow \pi_{1}(E) \rightarrow \pi_{1}(B) \rightarrow 1$, and this is $0 \rightarrow C \rightarrow G \rightarrow$ Aut $G$ $\rightarrow$ Out $G \rightarrow 1$.

2. The "action" of $\pi_{1}(B)$ on $\pi_{1}(K(G, 1))$ which is a homomorphism $\pi_{1}(B)$ $\rightarrow$ Out $G$ is the identity.

3. The twisted $k$-invariant for $B$ is $u=\Phi(U)$, as described in Theorem $\mathrm{C}$.

Since this is a fibration with fiber a $K(G, 1)$, there is a map $f: B$ $\rightarrow B A K(G, 1)$ which induces $p$.

THeOREM 3.2. The map $f$ is a homotopy equivalence, and the constructed bundle is the universal bundle.

Theorems $\mathrm{C}$ and $\mathrm{F}$ easily follow from 3.1 and 3.2.

Proof of 3.2. Since actions of $\pi_{1}$ (base) on the fiber are preserved under pullbacks, it follows from (2) that the action in the universal fibration is also the identity and that $f_{*}: \pi_{1}(B) \rightarrow \pi_{1}(B A K(G, 1))$ is an isomorphism. The map of long exact homotopy sequences induced by $f$ now shows $f$ induces an isomorphism in homotopy groups.

Geometric proof of Theorem E. A homomorphism $\phi: \Pi \rightarrow$ Out $G$ induces a map $\phi^{\prime}: K(\Pi, 1) \rightarrow K($ Out $G, 1)$ and using $\S 2$ we get the following diagram (where, as usual, we confuse the element $u \in H_{\phi}^{3}(K($ Out $G, 1) ;\{C\})$ and a map $u: K($ Out $G, 1) \rightarrow L_{\text {Aut } C}(C, 3)$ such that $u^{*}(v)=u($ given by 2.1 .2$)$ ):
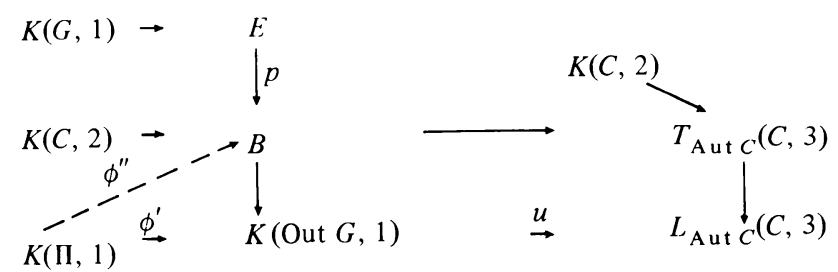

Let "the obstruction" be $\phi^{\prime *}(u)$. Then, as usual, the obstruction $=0$ iff there is a lifting $\phi^{\prime \prime}$ of $\phi^{\prime}$ (by 2.1.3) iff there is a $K(G, 1)$ fibration over $K(\Pi, 1)$ with "induced" action $\phi$ (this last follows since $p$ is the universal fibration, by using pullbacks, and since $\left.\phi=\phi_{*}^{\prime \prime}: \pi_{1}(K(\Pi, 1)) \rightarrow \pi_{1}(B)\right)$. If the obstruction is zero, i.e., if there is a $K(G, 1)$ bundle over $K(\Pi, 1)$ with "action" $\phi$, then $H_{\phi}^{2}(K(\Pi, 1) ;\{C\})$ is in $1-1$ correspondence with the set of homotopy classes of liftings $K(\Pi, 1) \rightarrow B$ of maps homotopic to $\phi^{\prime}$ (by 2.1.3) which is the set of homotopy classes of maps $K(\Pi, 1) \rightarrow B$ which induce the homomorphism $\phi: \pi_{1}(K(\Pi, 1)) \rightarrow \pi_{1}(B)$, which in turn is in $1-1$ correspondence with the equivalence classes of $K(G, 1)$ fibrations over $K(\Pi, 1)$ with "action" $\phi$.

4. We briefly recall the basic algebra we need.

The bar construction on $\Pi,\left\{B_{n}, \partial_{n}\right\}$, is a differential graded $\Pi$-module, where $B_{n}$ is the free Abelian gro'ı generated by all symbols of the form $x_{0}\left[x_{1}|\cdots| x_{n}\right]$, where $x_{i} \in \Pi$ and $x_{i} \neq 1$ if $i \geq 1$. See [9, pp. 114, 189]. If the Abelian group $C$ is a $\Pi$-module by $\gamma: \Pi \rightarrow$ Aut $C$, then get 


$$
\partial_{n}^{*}: \operatorname{Hom}_{Z(\Pi)}\left(B_{n-1}, C\right) \rightarrow \operatorname{Hom}_{Z(\Pi)}\left(B_{n}, C\right)
$$

and define $H_{\gamma}^{n}(K ; C)=\operatorname{ker} \partial_{n+1}^{*} / \operatorname{im} \partial_{n}^{*}$.

Let $G$ be a group, $C$ its center and $\alpha$ : Out $G \rightarrow$ Aut $C$ the natural homomorphism. Let $\phi: \Pi \rightarrow$ Out $G$ be a homomorphism. Consider the diagram

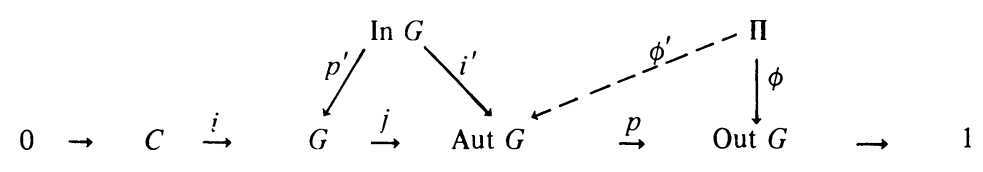

where $p$ and $p^{\prime}$ are the quotient homomorphisms, $i$ and $i^{\prime}$ are the inclusions, and $j=i^{\prime} p^{\prime}$ so that the row is exact. For each $x \in \Pi$, pick $\phi^{\prime}(x)$ $\in$ Aut $G$ so that $p \phi^{\prime}=\phi\left(\phi^{\prime}\right.$ is just a function!) but pick $\phi^{\prime}(1)=1$. Since $p\left[\phi^{\prime}(x) \phi^{\prime}(y) \phi^{\prime}(x y)^{-1}\right]=1$, for each $(x, y) \in \Pi \times \Pi$ we can pick $g(x, y) \in G$ such that $\phi^{\prime}(x) \phi^{\prime}(y)=j g(x, y) \phi^{\prime}(x y)$, but pick $g(1, y)=1=g(x, 1)$. The associative law in Aut $G$ gives $j\left[\phi^{\prime}(x)\{g(y, z)\} g(x, y z)\right]=j[g(x, y) g(x y, z)]$ so that for each $x, y, z \in \Pi$ there is a $K(x, y, z) \in C$ such that

$$
\phi^{\prime}(x)\{g(y, z)\} g(x y, z)=i K(x, y, z) g(x, y) g(x y, z) .
$$

Let $c \in \operatorname{Hom}_{Z(\Pi)}\left(B_{3}(\Pi), C\right)$ be given by

$$
c\left(x_{0}\left[x_{1}\left|x_{2}\right| x_{3}\right]\right)=\left\{\alpha \phi\left(x_{0}\right)\right\} K(x, y, z) .
$$

Then by Eilenberg and Mac Lane [4] (or see [9, pp. 126-128]):

THEOREM 4.4. The cochain $c$ is a cocycle and its cohomology class $\{c\}$ $\in H_{\alpha \phi}^{3}(\Pi ; C)$ is independent of the two choices made in the construction of $K$. There is an extension of $G$ by $\Pi$ which induces $\phi$ if and only if $\{c\}=0$.

Consider the special case $\Pi=$ Out $G$ and $\phi=$ id. We denote by

$$
\begin{aligned}
& v: \text { Out } G \rightarrow \text { Aut } G, \quad f: \text { Out } G \times \text { Out } G \rightarrow G, \\
& k: \text { Out } G \times \text { Out } G \times \text { Out } G \rightarrow C
\end{aligned}
$$

the functions constructed as above. We let $U \in H_{\alpha}^{3}($ Out $G ; C)$ be the corresponding cohomology class.

Theorem 4.6. This class $U \in H_{\alpha}^{3}$ (Out $G ; C$ ) is the universal example for this obstruction. I.e., given $\phi: \Pi \rightarrow$ Out $G$, then $\phi^{*}(U) \in H_{\alpha \phi}^{3}(\Pi ; C)$ is the obstruction to the existence of an extension of $G$ by $\Pi$ which induces $\phi$.

Proof. Simply let $\phi^{\prime}=v \phi, g=f(\phi \times \phi)$, and $K=k(\phi \times \phi \times \phi)$, verify the relations, and use 4.4 .

5. Description of the natural isomorphism $\Phi$. For a group $\Pi$, let $K(\Pi)$ be the standard Eilenberg-Mac Lane semisimplicial complex which is a Kan complex and is a $K(\Pi, 1)$. Its $n$-simplices are ordered $n$-tuples of elements of $\Pi$, $\left\langle a_{1}, \ldots, a_{n}\right\rangle$ for $n>0$, and a single 0 -simplex \langle\rangle . It has face and degeneracy operators 


$$
\begin{aligned}
\partial_{0}\left\langle a_{1}, \ldots, a_{n}\right\rangle & =\left\langle a_{2}, \ldots, a_{n}\right\rangle, \quad \partial_{n}\left\langle a_{1}, \ldots, a_{n}\right\rangle=\left\langle a_{1}, \ldots, a_{n-1}\right\rangle, \\
\partial_{i}\left\langle a_{1}, \ldots, a_{n}\right\rangle & =\left\langle a_{1}, \ldots, a_{i} a_{i+1}, \ldots, a_{n}\right\rangle, \quad 0<i<n, \\
s_{i}\left\langle a_{1}, \ldots, a_{n}\right\rangle & =\left\langle a_{1}, \ldots, a_{i}, 1, a_{i+1}, \ldots, a_{n}\right\rangle, \quad 0 \leq i \leq n .
\end{aligned}
$$

Let $B(\Pi)$ be the bar consturction on $\Pi$ (see $\S 4$ ), let $C$ be a $\Pi$-module, $c: B_{n}(\Pi) \rightarrow C$ an equivariant cocycle so that $\{c\} \in H_{\phi}^{n}(\Pi ; C)$.

LEMMA 5.1. The canonical isomorphism $\Phi: H_{\phi}^{*}(\Pi ; C) \rightarrow H_{\phi}^{*}(K(\Pi) ;\{C\})$ is given by $\Phi(\{c\})\left\langle a_{1}, \ldots, a_{n}\right\rangle=c\left(1\left[a_{1}|\cdots| a_{n}\right]\right)$.

Proof. Extend the argument given in [7, §3].

6. A construction of a certain Kan fibration. If $\alpha: \Pi \rightarrow G$ is a group homomorphism, then $\alpha^{\prime}: K(\Pi) \rightarrow K(G)$ by $\alpha^{\prime}\left\langle a_{1}, \ldots, a_{n}\right\rangle=\left\langle\alpha a_{1}, \ldots, \alpha a_{n}\right\rangle$ is a simplicial map and $\alpha_{*}^{\prime}: \pi_{1}(K(\Pi),\langle\rangle) \rightarrow \pi_{1}(K(G),\langle\rangle)$ is $\alpha$.

LEMmA 6.1. If $1 \rightarrow G \stackrel{i}{\rightarrow} E \stackrel{p}{\rightarrow} \Pi \rightarrow 1$ is exact, then $p^{\prime}: K(E) \rightarrow K(\Pi)$ is a Kan fibration with $i^{\prime}: K(G) \rightarrow K(E)$ the inclusion of the fiber.

Proof. This is well known and is just a checking of the definitions.

We will need an explicit description of $T=K(E)$ as a twisted cartesian product $K(G) \times{ }_{\tau} K(\Pi)$. The extension in 6.1 induces a homomorphism $\phi: \Pi \rightarrow$ Out $G$. Following $\S 4$, pick a function $\phi^{\prime}: \Pi \rightarrow$ Aut $G$ such that $p \phi^{\prime}=\phi, \phi^{\prime}(1)=1$. Since the obstruction is zero, we can pick a $g: \Pi \times \Pi$ $\rightarrow G$ such that $k(x, y, z)=0$, all $x, y, z \in \Pi$ (see [9, p. 127, 8.5]). Thus

$$
\phi^{\prime}(x)\{g(y, z)\} g(x, y z)=g(x, y) g(x y, z)
$$

(and $g(x, 1)=1=g(1, y))$. Then $T=K(G) \times K(\Pi)$ as a set with the definitions of $i^{\prime}, p^{\prime}, s_{i}$ for all $i$, and $\partial_{i}$ for $i>0$ obvious.

6.3. Define $\partial_{0}(a, b)=\left(\tau(b) \partial_{0} a, \partial_{0} b\right)$, where

$$
\left(\tau(b) \partial_{0} a\right)_{i}=\phi^{\prime}\left(b_{1}\right)^{-1}\left\{g\left(b_{1}, b_{2} \cdots b_{i-1}\right) a_{i} g\left(b_{1}, b_{2} \cdots b_{i}\right)^{-1}\right\},
$$

for $i=2, \ldots, n, a=\left\langle a_{1}, \ldots, a_{n}\right\rangle \in K(G)_{n}, b=\left\langle b_{1}, \ldots, b_{n}\right\rangle \in K(\Pi)_{n}$. Easily, with details left to the reader, $T$ is a semisimplicial complex and $K(G) \rightarrow T \rightarrow K(\Pi)$ is the required Kan fibration. We note that the relation $\partial_{0} \partial_{0}=\partial_{0} \partial_{1}$ is exactly where 6.2 is needed. (6.3 was obtained from $p^{\prime}$ by working backwards. See $[10, \S 19]$.)

7. Proof of 3.1. Let $U$ be as in Theorem B and $u=\Phi(U)$ (see 5.1). Let $K(C, 2) \rightarrow B \stackrel{q}{\rightarrow} K$ (Out $G)$ be the (pullback) twisted cartesian product with (twisted) $k$-invariant $u$ (see 3.3). So $B=K(C, 2) \times K\left(\right.$ Out $G$ ) as a set, $s_{i}$ all $i$ and $\partial_{i}$ for $i>0$ are clear. The twisting function for $B$ is induced by $u$ from that of $T_{\text {Aut } C}(C, 3)$ and hence from that of $L(C, 3)$, which we denote by $\tau_{1}$ and is given in May [10, p. 102 (ii)]. It is not hard to see that

$$
\partial_{0}(w, b)=\left(\alpha\left(b_{1}\right)^{-1}\left(\tau_{1}\left(b^{\prime *} u\right)+\partial_{0} w\right), \partial_{0} b\right)
$$

where $b=\left\langle b_{1}, \ldots, b_{n}\right\rangle, \alpha$ : Out $G \rightarrow$ Aut $C$ is the natural homomorphism, 
and $b^{\prime}: \Delta[n] \rightarrow K($ Out $G)$ is the simplicial map such that $b^{\prime}(0,1, \ldots, n)=b$ (so that $b^{\prime *}(u) \in Z^{3}(\Delta[n] ; C)$ ).

We build $E$ as a triply twisted cartesian product. As a set, $E=K(G) \times B$ $=K(G) \times K(C, 2) \times K($ Out $G)$. The maps $i: K(G) \rightarrow E, p: E \rightarrow B$, $s_{i}$ for all $i$, and $\partial_{i}$ for $i>0$ are all clear.

7.2. Define $\partial_{0}$ in $E$ by $\partial_{0}(a, w, b)=\left(\tau(b)\left(\sigma(w)+\partial_{0} a\right), \partial_{0}(w, b)\right)$. Here, $\partial_{0}(w, b)$ is as in 7.1, $\sigma$ is the twisting function for the fibration $K(C) \rightarrow L(C, 2)$ $\stackrel{\pi}{\rightarrow} K(C, 2),+: K(C) \times K(G) \rightarrow K(G)$ is induced by multiplication $C \times G$ $\rightarrow G$, and $\tau(b)$ ( ) is essentially the same $\tau$ given by (6.3) except that the $g$ and $\phi^{\prime}$ are replaced with the $f$ and $v$ of 4.4 .

It will now follow that $p$ is a Kan fibration and that $E$ is a Kan complex. Verifying the relation $\partial_{0} \partial_{0}=\partial_{0} \partial_{1}$ is a little involved and is exactly where (4.2) is needed. One step is to observe for 3 -simplices $(0,1,2, j) \in \Delta[n]$, $b^{\prime}(0,1,2, j)=\left\langle b_{1}, b_{2}, b_{3}, \ldots, b_{j}\right\rangle$, so that

$$
\begin{aligned}
b^{\prime *}(u)(0,1,2, j) & =u\left\langle b_{1}, b_{2}, b_{3} \cdots b_{j}\right\rangle=U\left[b_{1}\left|b_{2}\right| b_{3} \cdots b_{j}\right] \text { by } 5.1 \\
& =k\left(b_{1}, b_{2}, b_{3} \cdots b_{j}\right) \text { by } 4.3 .
\end{aligned}
$$

Remaining details are left to the reader.

Proof of 3.1.3. Immediate by the construction.

Proof of 3.1.1. By construction, $\pi_{1}(B)=$ Out $G, \pi_{2}(B)=C$, and $\pi_{i}(B)$ $=0$ otherwise. Using the construction of $E$, it is easy to construct a map of fibrations from $\pi$ to $p$ such that $K(C) \rightarrow K(G)$ and $K(C, 2) \rightarrow B$ are both the natural inclusions. Naturality now yields that $\partial: \pi_{2}(B) \rightarrow \pi_{1}(K(G))$ is the inclusion $C \rightarrow G$.

The extension In $G \stackrel{i^{\prime}}{\rightarrow}$ Aut $G \stackrel{q}{\rightarrow}$ Out $G$ induces a $\phi:$ Out $G \rightarrow$ Out (In $G$ ). From diagram (4.1) we get the following diagram

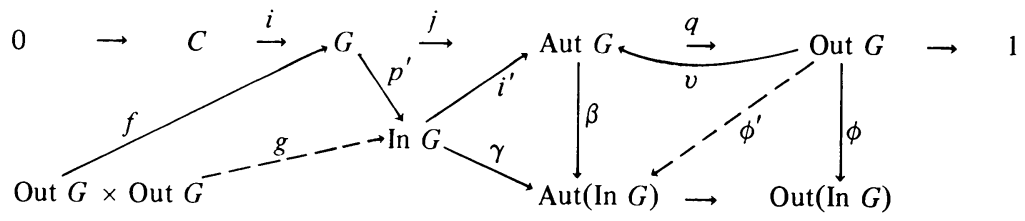

where $v$ and $f$ are given in 4.4. Let $\phi^{\prime}=\beta v$ and $g=p^{\prime} f$, and construct the Kan fibration $K(\operatorname{In} G) \rightarrow K($ Aut $G, 1) \rightarrow K($ Out $G)$ as in $\S 6$. From the explicit twisting functions given, we get a map of fibrations

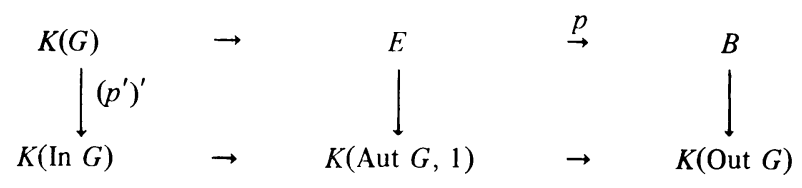

Using the induced map of homotopy sequences and the fact that $\left(p^{\prime}\right)_{*}^{\prime}$ $=p^{\prime}$ on $\pi_{1}$, the rest of 3.1.1 follows.

Proof OF 3.1.2. Let $S^{1}=\Delta[1] /\{(0),(1)\}$ and identify the 1-cell $[a] \in K(G)$ with the map $S^{1} \rightarrow K(G)$ generated by $(0,1) \rightarrow[a]$. Then the isomorphism $G \cong \pi_{1}(K(G), *) \quad(*=[1]=$ the only 0 -cell $) \quad$ is induced by $a \leftrightarrow[a]$. Similarly for $K($ Out $G)$ and for $B=K(C, 2) \times{ }_{\rho} K($ Out $G)$. Pick $a \in G$ and $b$ 
$\in$ Out $G$. To prove 3.1.2, it is sufficient to find a homotopy $S^{1} \times I \rightarrow E$ which starts with $([a], *, *)$, ends with $\left(\left[b^{-1}\{a\}\right], *, *\right)$ and which lies over $\left(*,\left[b^{-1}\right]\right)$ $\in B$. Using May [10, 5.1 and 6.2], it is sufficient to exhibit two 2-cells of $E$ which match up along a common boundary and which satisfy the conditions indicated in the diagram

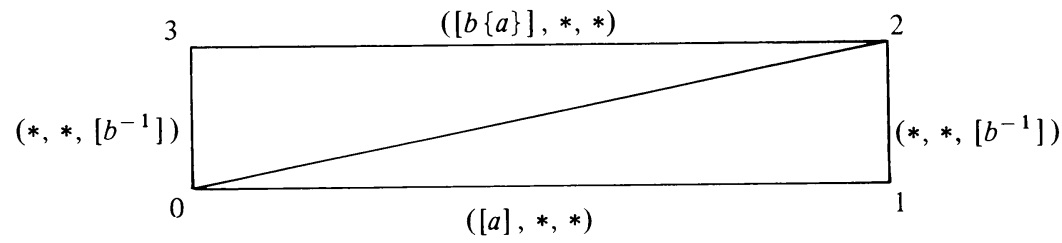

Letting $\langle 0,1,2\rangle=\left([a \mid 1], *,\left[1 \mid b^{-1}\right]\right)$ and $\langle 0,2,3\rangle=\left([1 \mid a], *,\left[b^{-1} \mid 1\right]\right)$ (note that $\left.\partial_{0}\langle 0,2,3\rangle=\left(\left[\left(b^{-1}\right)^{-1}\{a\}\right], *,[1]\right)\right)$ completes the proof.

\section{BIBLIOGRAPHY}

1. M. G. Barratt, V. K. A. M. Gugenheim and J. C. Moore, On semisimplicial fibre-bundles, Amer. J. Math. 81 (1959), 639-657. MR 22 \#1895.

2. A. Bousfield and D. M. Kan, Homotopy limits, completions, and localizations, Lecture Notes in Math., vol. 304, Springer-Verlag, Berlin and New York, 1972.

3. S. Eilenberg, Homology of spaces with operators. I, Trans. Amer. Math. Soc. 61 (1947), 378-417; errata, ibid. 62 (1947), 548. MR 9, 52.

4. S. Eilenberg and S. Mac Lane, Cohomology theory in abstract groups. I, II, Ann. of Math. (2) 48 (1947), 51-78, 326-341. MR 8, 367; 9, 7.

5. S. Gitler, Cohomology operations with local coefficients, Amer. J. Math. 85 (1963), 156-188. MR 28 \#1621.

6. D. H. Gottlieb, On fibre spaces and the evaluation map, Ann. of Math. (2) 87 (1968), 42-55; correction, ibid. (2) 91 (1970), 640-642. MR 36 \#4560; 41 \#7684.

7. R. O. Hill, Jr., On characteristic classes of groups and bundles of $K(\Pi, 1)$ 's, Proc. Amer. Math. Soc. 40 (1973), 597-603. MR 47 \#737.

8. J. F. McClendon, Obstruction theory in fiber spaces, Math. Z. 120 (1971), 1-17. MR 45 \#6002.

9. S. Mac Lane, Homology, Die Grundlehren der math. Wissenschaften, Band 114, Academic Press, New York; Springer-Verlag, Berlin, 1963. MR 28 \#122.

10. J. P. May, Simplicial objects in algebraic topology, Van Nostrand Math. Studies, no. 11, Van Nostrand, Princeton, N. J., 1967. MR 36 \# 5942.

11. F. Nussbaum, Thesis, Northwestern University, 1970.

12. - - Semi-principal bundles and stable nonorientable obstruction theory (to appear).

13. P. Olum, On mappings into spaces in which certain homotopy groups vanish, Ann. of Math

(2) 57 (1953), 561-574. MR 14, 895.

14. - Factorizations and induced homomorphisms, Advances in Math. 3 (1969), 72-100. MR 38 \#6590.

15. J. Siegal, Higher order cohomology operations in local coefficient theory, Amer. J. Math. 89 (1967), 909-931. MR 37 \#913.

16. —, k-invariants in local coefficient theory, Proc. Amer. Math. Soc 29 (1971), 169-174. MR 46 \#6344.

17. C. Robinson, Moore-Postnikov systems for non-simple fibrations, Illinois J. Math. 16 (1972), 234-242.

Department of Mathematics, Michigan State University, East Lansing, Michigan 48824 